\title{
Residual Stress Measurement on a MEMS Structure With High-Spatial Resolution
}

\author{
Neus Sabaté, Dietmar Vogel, Astrid Gollhardt, Jürgen Keller, Carles Cané, Isabel Gràcia, Joan R. Morante, and
} Bernd Michel

\begin{abstract}
A new approach to the local measurement of residual stress in microstructures is described in this paper. The presented technique takes advantage of the combined milling-imaging features of a focused ion beam (FIB) equipment to scale down the widely known hole drilling method. This method consists of drilling a small hole in a solid with inherent residual stresses and measuring the strains/displacements caused by the local stress release, that takes place around the hole. In the presented case, the displacements caused by the milling are determined by applying digital image correlation (DIC) techniques to high resolution micrographs taken before and after the milling process. The residual stress value is then obtained by fitting the measured displacements to the analytical solution of the displacement fields. The feasibility of this approach has been demonstrated on a micromachined silicon nitride membrane showing that this method has high potential for applications in the field of mechanical characterization of micro/nanoelectromechanical systems.

[1634]
\end{abstract}

Index Terms-Microreliability, residual stress.

\section{INTRODUCTION}

$\mathbf{M}$ EASUREMENT of residual stresses is an important requirement for micro/nanoelectromechanical systems (MEMS/NEMS) development. Sometimes, the resulting stresses can induce a variety of consequences which are unfavorable in most circumstances-excessive deformation, fracture, delamination, and microstructural changes in the material that can lead to breaking of structures during fabrication or affect the behavior of the final device as well as its reliability [1], [2]. Consequently, the control of residual stress states is a key issue to reliability of future products. Because of the persistently increasing miniaturization and complexity of microsystem devices, there will be a strong need for development of advanced residual stress measurement tools in the next years.

When downscaling to micronano structures, classical methods of material testing are often impossible because appropriate testing specimens cannot be manufactured in an

Manuscript received June 22, 2005; revised September 1, 2005. Subject Editor S. M. Spearing.

N. Sabaté is with the Micro Materials Centre, Fraunhofer IZM Berlin, 13355 Berlin, Germany. She is also with the Departament d'Electrònica, Universitat de Barcelona (EME-UB), 08028, Barcelona, Spain (e-mail: Neus.Sabate@izm. fhg.de).

D. Vogel, A. Gollhardt, J. Keller, and B. Michel are with the Micro Materials Centre, Fraunhofer IZM Berlin, 13355 Berlin, Germany (e-mail: dietmar. vogel@izm.fraunhofer.de).

C. Cané and I. Gràcia are with the Centre Nacional de Microelectrònica, (CNM-CSIC), E-08193, Bellaterra, Barcelona, Spain.

J. R. Morante is with the Departament d'Electrònica, Universitat de Barcelona (EME-UB), 08028, Barcelona, Spain.

Digital Object Identifier 10.1109/JMEMS.2006.879701 efficient way. As a consequence, new mechanical testing approaches must be able to measure displacements, strains, and forces within completely shifted measurement ranges. In the last decade, several attempts to do so have been developed: methods like the curvature measurements at wafer level or the bulge based tests have become common ways to obtain stresses in thin films [2]-[4]. Other approaches have focused on the on-purpose fabrication of micromachined structures with suitable geometries such as rings or cantilevers [5]-[7]. However, even if these methods have proved to provide acceptable measurements of the residual stress, none of them is able to provide local stress measurement with high spatial resolution.

In this paper, we present a new approach to the measurement of residual stress in MEMS structures with high-local spatial resolution. This method uses the combined imaging-milling features of a FIB equipment to scale down to the microscale the widely known hole-drilling method. This method consists of the release of residual stresses by material removal and subsequent measurement of the induced strains in the near region of the removed volume. In this way, through or blind holes are milled into the layer under study and induced strains are measured by strain gages placed at determined positions [8]. Unfortunately, mechanical or laser based material removal is restricted in size and so it is the placement of adequate strain gages on the surface of a small structure. In this sense, strain measurement techniques by interferometry [i.e., electronic speckle pattern interferometry (EPSI) technique] eliminated to a certain extend the need and thereby, the mechanical problems associated with the downscaling of strain gauging [9]. However, the extension of the hole-drilling method to a micron range requires the use of alternative tools. In this sense, two major interesting approaches have been made to transfer interferometric strain measurement principles to higher magnification microscopes like SEM or AFM. The first approach hinges on Moire techniques [10], [11], whereas the second is based on the use of speckle techniques [12]. Very recently, the hole-drilling method principle has been applied to assess the elastic properties of a sample. The approach measures the displacements occurred around the hole by applying digital image correlation analysis on AFM images [13], [14].

In the present work, a FIB cross-beam equipment has been used to mill the stress-release features on a silicon nitride micromachined membrane. Micrographs of the milled area before and after the stress release have been captured by the scanning electron column. Displacements originated from the milling process have been measured by applying digital image correlation (DIC) techniques to the captured high resolution micrographs. Finally, 
the residual stress value is then obtained from the fitting of the measured displacements to the analytical solution of the displacement fields. With regard to FIB applications, this method has been established by the authors under the name fibDAC [15].

\section{Hole-Drilling TeChniQue on a Microscale}

The hole-drilling Strain Gage Method is one of the most widely used methods for determining residual stresses and it is based on the measurement of local stress relaxation at the sample under study. That is, the introduction of a hole into a material containing residual stresses results in an immediate stress relaxation at the free surfaces of the milled feature that also changes the stress distribution in the surrounding region, causing the local strains on the surface of the test material to change correspondingly. The common procedure for measuring the resulting strains is to mount three resistance strain gages in the form of a rosette around the site of the hole before drilling by air turbine and carbide cutters, steel twist drills or laser based material removal techniques [16].

However, when considering a downscaling of this method of residual stress measurement in a MEMS structure, mechanical or laser based material removal encounter size restrictions and so it happens with the placement of adequate strain gages. In this sense focused ion beam (FIB) instruments arise as a very suitable tool that allows to extend the hole-drilling method deeply into the submicron range. FIB equipment permits the milling of features down to the micrometer and nanometer scale and allow at the same time micro/nanoscopic inspection of the sample under consideration. Taking advantage of these unique features, FIB equipment can be used to mill the stress-release features on MEMS structures. Micrographs of the milled area before and after the stress release are captured by a scanning electron column, which is part of the FIB equipment. In this way, it is not the strains (as with the macroscopic technique) but primarily the displacements originating from the milling process that can be measured applying the digital image correlation (DIC) technique to the captured high resolution micrographs.

In previous research the authors developed and modified special equipment in order to apply scanning electron microscopy for deformation analysis on thermomechanically loaded electronic packages. The respective technique was established as micro Deformation Analysis by means of Correlation algorithms (microDAC) [17] and it is a specialized DIC application [18]. Generally, images extracted from a variety of sources such as SEM or laser scanning microscopy (LSM) can be utilized for the application of digital cross correlation.

In this technique, a complete set of local image patterns coming from the digital images are correlated. The result of a two-dimensional (2-D) cross correlation with subpixel analysis in the surroundings of a correlation point primarily gives the two components of the in-plane displacement vector. Applied to a set of correlation points (e.g., to a rectangular grid of points within a user defined pitch), this method allows to extract the complete in-plane displacement field.

The accuracy of the determined displacement values in the field is defined by the micrograph size in absolute coordinate values $L_{x, y}$, the image pixel size $M_{x, y}$, and the subpixel resolution of the refinement method strategy $\mathrm{k}$, a value that for images with moderate decorrelation of patterns can be in between 0.05 and 0.1

$$
\delta u_{\mathrm{x}, \mathrm{y}}=\left(\frac{L_{\mathrm{x}, \mathrm{y}}}{M_{\mathrm{x}, \mathrm{y}}}\right) k=N_{\mathrm{x}, \mathrm{y}} \cdot k .
$$

Due to its nature, the method possesses an excellent downscaling capability that makes it specially suitable for our purposes. Displacement measurement accuracy scales with the image size L, [see (1)] so applying the same DIC software algorithms to images with higher magnification allows not only to improve lateral position resolution, but also the measurement accuracy itself.

\section{A. Through-Hole Milling on a Prestressed Plate}

In most practical applications, the drilled hole is blind but unfortunately, no closed-form solution is available from the theory of elasticity for direct calculation of residual stresses from the measured strains-except by the introduction of empirical coefficients. An exact solution can be obtained, however, for the simpler case of a hole drilled completely through a thin plate in which the residual stress is uniformly distributed through the plate thickness [19]. As a way to demonstrate the feasibility of this technique, only the through-hole case has been considered in this paper. However, the present procedure can be used to mill geometries with no-analytical solution. In that case, the displacements caused by the stress release can be modeled with Finite Element Simulations.

Assuming a homogeneous uniaxial stress $\sigma_{x x}$ and plane stress state conditions, the strain caused by the milling of a through hole of radius $R_{0}$ in an homogeneous material with elastic behavior can be expressed as

$$
\begin{gathered}
\varepsilon_{\theta}=-\frac{\sigma_{x x}(1+\nu)}{2 E}\left[-\frac{R_{0}^{2}}{r^{2}}-\frac{3 R_{0}^{4}}{r^{4}} \cos 2 \theta-\frac{4 R_{0}^{2} \nu}{r^{2}(1+\nu)} \cos 2 \theta\right] \\
\varepsilon_{\mathrm{r}}=-\frac{\sigma_{x x}(1+\nu)}{2 E}\left[\frac{R_{0}^{2}}{r^{2}}-\frac{3 R_{0}^{4}}{r^{4}} \cos 2 \theta+\frac{4 R_{0}^{2}}{r^{2}(1+\nu)} \cos 2 \theta\right] .
\end{gathered}
$$

E being Young's modulus and $\nu$ Poisson ratio of the sample and $\theta$ and $\mathrm{r}$ representing the coordinates in a cylindrical coordinate system with its origin in the center of the milled hole. Integration of the corresponding strains generated in the stress release under appropriate boundary conditions (i.e., $\mathrm{u}_{\mathrm{r}}(\infty, \theta)=0$ and $\mathrm{u}_{\theta}(\mathrm{r}, 0)=0$ ) and transformation of the solution to the Cartesian coordinate system leads to the following expressions of the displacement field

$$
\begin{aligned}
u_{\mathrm{x}}= & -\frac{\sigma_{x x}(1+\nu)}{2 E} \\
& \cdot\left[-\frac{R_{0}^{2}}{r}\left(\frac{4}{(1+\nu)} \cos \theta+\cos (3 \theta)\right)+\frac{R_{0}^{4}}{r^{3}} \cos (3 \theta)\right] \\
u_{\mathrm{y}}= & -\frac{\sigma_{x x}(1+\nu)}{2 E} \sin \theta \\
& \cdot\left[\frac{R_{0}^{2}}{r}\left(\frac{(3-\nu)}{(1+\nu)}-4 \cos ^{2} \theta\right)+\frac{R_{0}^{4}}{r^{3}}\left(-1+4 \cos ^{2} \theta\right)\right] .
\end{aligned}
$$




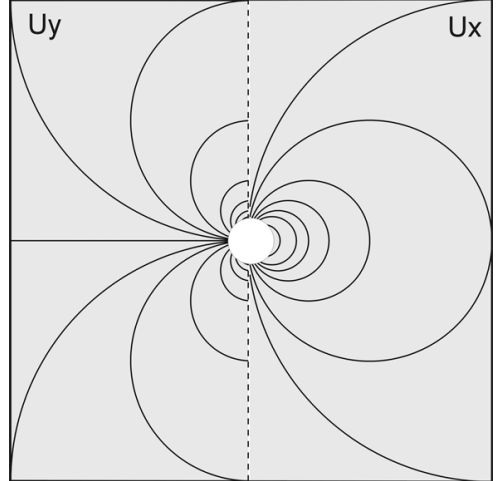

Fig. 1. Contour lines of the analytical displacement fields $\mathbf{u}_{\mathbf{x}}$ and $\mathbf{u}_{\mathrm{y}}$ corresponding to a generic solution of an homogeneous biaxial prestressed material.

The mentioned integration has been performed for the complete differentials $d u_{\mathrm{r}}$ and $d u_{\theta}$ of the pure elastic problem over a properly chosen integration path. A definite integral from infinity to $r$ along a constant angle $\theta$ (so the summand $\int_{\theta_{1}}^{\theta_{2}}\left(\partial u_{\mathrm{r}} / \partial \theta\right) d \theta$ for $\theta_{1}=\theta_{2}$ can be omitted) was chosen for the derivation of $\mathrm{u}_{\mathrm{r}}(\mathrm{r}, \theta)$. The reasonable boundary condition $\mathrm{u}_{\mathrm{r}}(\infty, \theta)=0$ was used. The $\mathrm{u}_{\theta}(\mathrm{r}, \theta)$ derivation was based on a definite integral of $\mathrm{du}_{\theta}$, where the integration path has been chosen along a circle with constant radius. In this case the boundary condition $\mathrm{u}_{\theta}(\mathrm{r}, 0)=0$ has been exploited, which follows from the axi-symmetry of the mechanical problem with regard to the line $\mathrm{y}=0$.

The values $\mathrm{u}_{\mathrm{x}}$ and $\mathrm{u}_{\mathrm{y}}$ in (4) and (5) can be directly measured by the image correlation. In the case of a biaxial residual stress, a $90^{\circ}$ rotated solution of the displacement field with a residual stress intensity of $\sigma_{\mathrm{yy}}$ must be superimposed on the solution given by (4) and (5). Under the presence of an homogeneous biaxial stress state, that is, $\sigma_{x x}=\sigma_{\mathrm{yy}=} \sigma_{0}$, expressions for the displacement field simplify in a very convenient way

$$
\begin{aligned}
& u_{\mathrm{x}}(\mathrm{r}, \theta)=\frac{\sigma_{0} \mathrm{R}_{0}^{2}}{\mathrm{E} \mathrm{r}}(1+\nu) \cos \theta \\
& \mathrm{u}_{\mathrm{y}}(\mathrm{r}, \theta)=\frac{\sigma_{0} \mathrm{R}_{0}^{2}}{\mathrm{E} \mathrm{r}}(1+\nu) \sin \theta
\end{aligned}
$$

that leads to a circular symmetry for the displacement modulus

$$
|\mathrm{u}|=\frac{\sigma_{0} \mathrm{R}_{0}^{2}}{\mathrm{E} \mathrm{r}}(1+\nu) \text {. }
$$

Fig. 1 shows the contour lines of the analytical displacement fields $\mathrm{u}_{\mathrm{x}}$ and $\mathrm{u}_{\mathrm{y}}$ corresponding to a generic solution of a homogeneous biaxial prestressed material. Due to the assumed isotropy on the stress distribution, the corresponding displacement components are symmetric with respect to the $\mathrm{x}$ and $\mathrm{y}$ axes. The higher displacements concentrate in the region near the hole and extend up to a certain distance.

According to (8), the extent of the stress release region along a certain radius varies from $\sigma_{\mathrm{r}}=0$ at the edge of the hole to the remote stress value $\sigma_{0}$ according to an inverse square law

$$
\sigma_{\mathrm{r}}=\sigma_{o}\left(1-\frac{R_{o}^{2}}{r^{2}}\right) \text {. }
$$

Expression (9) shows that the stress distribution approaches quickly the magnitude of the remote stress at a short distance

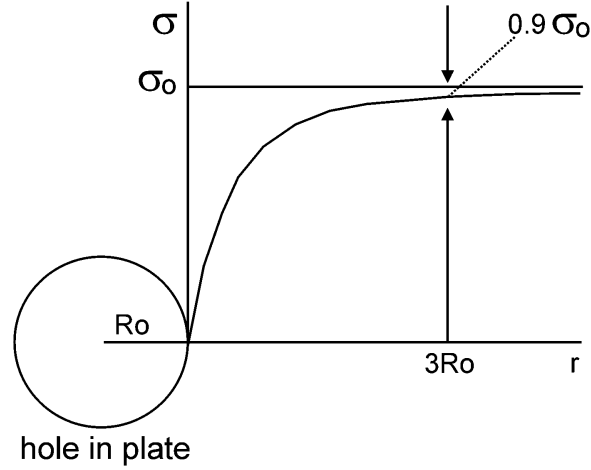

Fig. 2. Evolution of the stress along an arbitrary radius for a biaxial loaded plate with a circular milled hole at the center.

from the hole boundary. The effect of the hole on the stress distribution is therefore, localized to the region around it. In the studied case (i.e., through-hole milling) the stress field, which drops to zero at the boundary of the hole due to the traction-free conditions, returns to a value of the $90 \%$ of the biaxial uniform field $\sigma_{0}$ at a distance of approximately 3 hole radii (see Fig. 2). From (9) we note that the distribution of stress depends on the normalized distance $r / R_{0}$ and, consequently, the affected stress region scales with $\mathrm{R}_{0}$, i.e., the hole size. Therefore, the spatial resolution of this method is directly related to the radius of the hole milled in the layer under study.

\section{B. Accuracy of the DIC Method}

The accuracy of residual stress measurement is limited in this particular case by the accuracy of the displacement field measurement as well as by the accuracies of the elastic modulus and Poisson ratio.

Whereas the accuracy of these last two material parameters depends on the technique used to characterize them, the accuracy on the displacement measurements is related to the magnification of the captured image [see (1)]. So theoretically, the accuracy could be increased until any desired value by decreasing the size of the scanned area until reaching the resolution of the electron imaging column. However, in order to be able to compare with the corresponding analytical solution, it seems reasonable to keep the minimum vertical size of the scanned image to $2 \mathrm{R}_{0}$, being $\mathrm{R}_{0}$ the milled hole radius. Taking this criterion into account the displacement accuracy is set to

$$
\delta u=\frac{2 R_{0}}{M} k
$$

with $\mathrm{M}$ as the number of image pixels and $\mathrm{k}$ as the subpixel resolution.

On the other hand, accuracy requirements when measuring displacements are strongly related to the magnitude of these displacements. As set in (8) displacement caused by milling has a square dependence on the hole radius Ro, which yields the following dependence of relative error

$$
\frac{\delta u}{u}=\frac{2 k E}{M \sigma(1+\nu)\left(\frac{R_{0}}{r}\right)} .
$$

So, independently from the magnification, the relative error of our measurements in the range of $r=R_{0}$ and $r=2 R_{0}$ will 


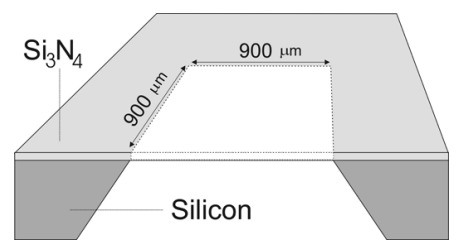

Fig. 3. Scheme of the silicon chip tested in this work (cross section). The central squared zone corresponds to the 300-nm silicon nitride micromachined membrane.

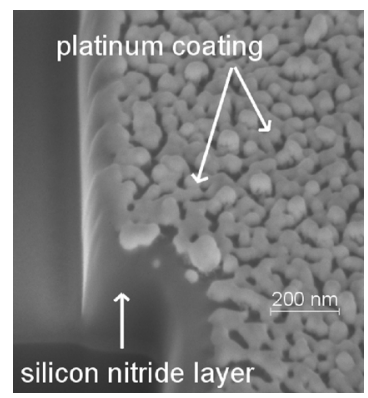

Fig. 4. High-magnification SEM image of a membrane cross section. The thin layer of patterned platinum on top of the silicon nitride layer can be clearly distinguished.

always yield the same value. Replacing $M$ in (11) by a value of 1000 and $\mathrm{k}$ by 0.1 we get an estimate of $\delta \mathrm{u} / \mathrm{u} \approx 2 \cdot 10^{-4} \mathrm{E} / \sigma$. Assuming for $\mathrm{E} / \sigma$ a value of 500 for maximum residual stresses -because of elasticity limits of materials- $\delta \mathrm{u} / \mathrm{u}$ reaches a value of 0.1 , that is, a measurement error of $10 \%$ for a single measurement point is to be considered. As a consequence, even for high residual stresses, stress determination from a two point measurement is not efficient. Whole field comparison between theoretical and measured displacement fields based on better statistics is needed to gain sufficient accuracy in the determination of stress values.

\section{EXPERIMENTAL}

The feasibility of this new technique has been tested on silicon nitride micromachined membranes. This kind of structure plays an important role as mechanical support in a wide range of membrane-based sensors [20], [21]. The layer under study was a 300-nm-thick low-pressure chemical vapor depositioned (LPCVD) silicon nitride membrane. The deposition was performed onto a silicon substrate at $800{ }^{\circ} \mathrm{C}$ under a residual pressure in the range of 190-210 mtorr. Sample processing ended with the silicon micromachining of the substrate, which was performed by means of a back side $\mathrm{KOH}$ etching. A scheme of the micromachined structure under investigation is shown in Fig. 3. To make the image correlation possible, the surface of the object has to be provided with a certain pattern. In the present case, the 20-nm-thick platinum flash coating deposited on the nitride surface in order to avoid surface charging during SEM imaging fulfilled this demand. In Fig. 4, a SEM cross section performed on one of the samples that shows the deposited platinum layer on top of the silicon nitride is depicted. Due to the configuration of this layer in islands, no significant effect on the total mechanical behavior of the layer under study is expected.

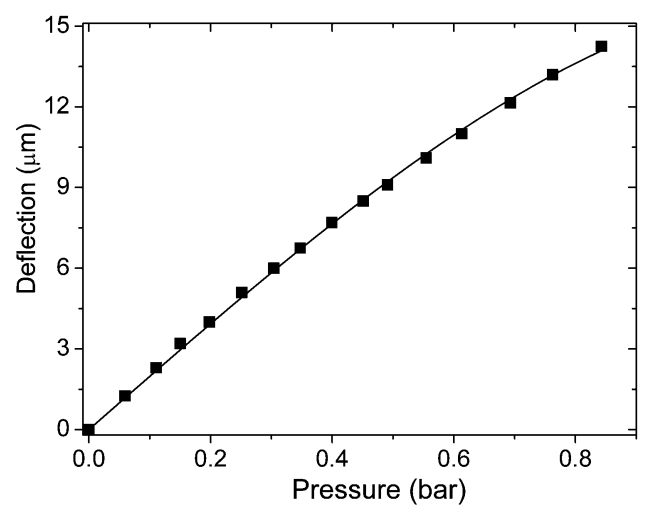

Fig. 5. Results obtained with the bulge test: out-of-plane deformation at the membrane center versus applied pressure.

\section{A. Layer Characterization With Standard Techniques}

In order to obtain reference values of Young modulus and residual stress to compare with those obtained in the holedrilling method, the residual stress and the Young modulus of the 300-nm-thick nitride layer were measured with standard methods. A bulge test was performed to bare membranes (see Fig. 3). This method is particularly well suited in the present case because it allows to determine the residual stress of the samples as processed. Young modulus was accurately determined by nanoindentation tests.

An homogeneous air pressure was applied at the lower side on the membrane and the resulting deflection was measured by means of a microinterferometer. The deflection in the middle of the membrane is a function of its elastic material properties and of the residual stress. Two different samples of each kind were measured and showed good reproducibility within measurement error (Fig. 5 shows the average value of deflection at each pressure). Fringes of $0.275 \mu \mathrm{m}$ deflection steps were counted. Error in measurement was considered as $\pm 1 / 2$ fringe. Compared with errors for the membrane deflection, errors in pressure are considered negligible, as accuracy in pressure was $1 \%$ FSO. As a way of introducing error in deflection values of the fitting calculations, data was scattered $\pm 1 / 2 * 0.275$. The dependence of the central point of the membrane versus pressure was taken from the general expression for squared membranes in large deflection regime [22]

$$
p=\frac{C_{1} \sigma t}{a^{2}} h+\frac{C_{2}(\nu) E t}{a^{4}} h^{3}
$$

where $p$ is the applied pressure, $t$ is the membrane thickness, $a$ is one half of the membrane's edge length, $E$ is the Young's modulus, $\sigma$ is the residual stress, $\nu$ is the in-plane Poisson's ratio, and $h$ is the deflection at the center of the membrane.

In the present case, membranes had $900 \mu \mathrm{m}$ side length. Values of constants $\mathrm{C}_{1}$ and $\mathrm{C}_{2}$ were taken from the solution proposed by Maier-Schneider et al. [23], where $\mathrm{C}_{1}$ is 3.45 and $\mathrm{C}_{2}$ is set to 2.43 for a Poisson ratio of 0.23 , a value commonly taken as initial design value for silicon nitride [3]. Fig. 5 shows the out-of-plane deformation measured at different pressures values. Data was fitted to (12) within a factor of $\mathrm{R}^{2}=0.998$ and yielded a residual stress of $1010 \pm 20 \mathrm{MPa}$ and a Young modulus of $214 \pm 25 \mathrm{GPa}$. The value for the Young modulus 


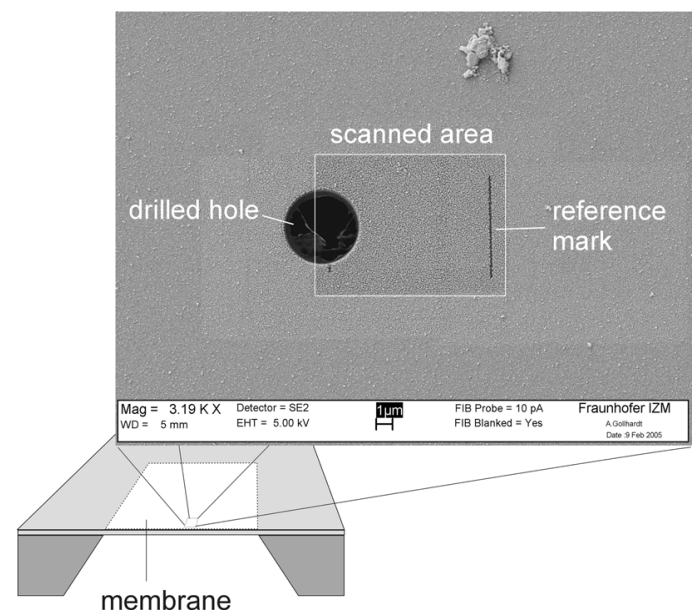

Fig. 6. Scheme of a micromachined membrane and general view of the scanned area after the hole-milling process.

is in agreement with the one determined independently by nanoindentation measurements; measured values for different indentation depths yielded a value of $224 \pm 11 \mathrm{GPa}$. This value corresponds to a extrapolation of different measured curves to a zero indentation depth [24].

\section{B. fibDAC Measurement}

In our experiment, the sample was placed in a Zeiss $1540 \mathrm{XB}$ Crossbeam equipment and a hole of $4.5 \mu \mathrm{m}$ was milled at the central area of one of the high stressed nitride membranes. SEM imaging of a small area placed at the middle of the membrane was performed at $10 \mathrm{~K}$ magnification. Measurement accuracy for displacements was nearby $1 \mathrm{~nm}$, a value given for a pixel size of $10.9 \mathrm{~nm}$ and a subpixel resolution of approximately 0.1 . Fig. 6 shows a general view of the scanned area after the holedrilling process. Due to the different orientation of the scanning electron and the ion beam columns, the sample needs to be tilted $54^{\circ}$ between the imaging and the milling steps. A superficial (between 10 and $20 \mathrm{~nm}$ ) vertical line at the right side of the image was used as reference mark between the steps, in order to reposition SEM images after ion milling.

After performing image correlation analysis to the images obtained with FIB equipment before and after the milling process, results are stored in a data file that contains information about the displacement field $\mathrm{u}_{\mathrm{x}}(\mathrm{x}, \mathrm{y})$ and $\mathrm{u}_{\mathrm{y}}(\mathrm{x}, \mathrm{y}), \mathrm{x}, \mathrm{y}$ being the position in pixels of a particular displacement in the analyzed image. Fig. 7(a) and (b) shows the experimental contour lines for $u_{x}$ and $\mathrm{u}_{\mathrm{y}}$, respectively. For clarity, data has been superimposed on one of the SEM images used in the correlation analysis. The experimental results have to be fitted now to the analytical expressions given in (6) and (7). Fitting has been performed for the whole available 2-D displacement field. This approach is necessary because of the accuracy considerations aforementioned in Section II-B. The statistics of the whole field allows to improve significantly the achieved final accuracy for residual stress values.

Before starting the fitting process, it has to be taken into account that some additional displacements coming from a rotation of the sample between the two different image capture

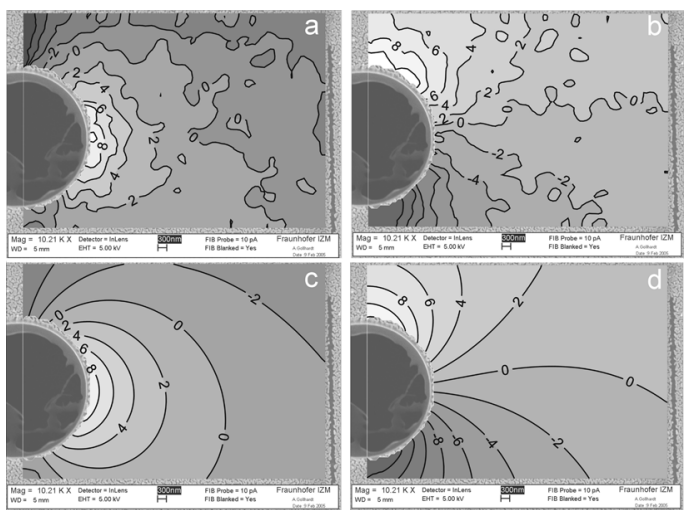

Fig. 7. (a) $\mathbf{u}_{\mathrm{x}}$ component of the experimental displacement field. (b) $\mathbf{u}_{\mathrm{y}}$ component of the experimental displacement field. (c) $\mathbf{u}_{\mathrm{x}}$ analytical best fitting. (d) $\mathbf{u}_{\mathrm{y}}$ analytical best fitting. All data is in nanometers.

shots could be superposed to the displacement field caused by the milling process. In order to include these displacements, an arbitrary rotation $\alpha$ and a rigid body displacement $\mathrm{x}_{0}, \mathrm{y}_{0}$ have been introduced in the fitting process as will be explained in the following.

The milling displacement field (without rotation effects) at every point can be defined as

$$
\begin{aligned}
& u_{x}(x, y)=x_{f}-x \\
& u_{y}(x, y)=y_{f}-y .
\end{aligned}
$$

$\mathrm{x}_{\mathrm{f}}$ and $\mathrm{y}_{\mathrm{f}}$ being the final position of every measured point. On the other hand, a rotation between the initial and the final images would yield a slightly changed displacement field that can be expressed as

$$
\begin{aligned}
& \mathrm{u}_{\mathrm{x}}^{\prime}(\mathrm{x}, \mathrm{y})=\mathrm{x}_{\mathrm{f}}^{\prime}-\mathrm{x} \\
& \mathrm{u}_{\mathrm{y}}^{\prime}(\mathrm{x}, \mathrm{y})=\mathrm{y}_{\mathrm{f}}^{\prime}-\mathrm{y}
\end{aligned}
$$

where

$$
\begin{aligned}
& \mathrm{x}_{\mathrm{f}}^{\prime}=\mathrm{x}_{\mathrm{f}} \cos \alpha+\mathrm{y}_{\mathrm{f}} \sin \alpha+\mathrm{x}_{0} \\
& \mathrm{y}_{\mathrm{f}}^{\prime}=-\mathrm{x}_{\mathrm{f}} \sin \alpha+\mathrm{y}_{\mathrm{f}} \cos \alpha+\mathrm{y}_{0} .
\end{aligned}
$$

So the displacement field measured by fibDAC if a rotation has occurred corresponds to

$$
\begin{aligned}
& \mathrm{u}_{\mathrm{x}}^{\prime}(\mathrm{x}, \mathrm{y})=\mathrm{x}_{\mathrm{f}} \cos \alpha+\mathrm{y}_{\mathrm{f}} \sin \alpha+\mathrm{x}_{0}-\mathrm{x} \\
& \mathrm{u}_{\mathrm{y}}^{\prime}(\mathrm{x}, \mathrm{y})=-\mathrm{x}_{\mathrm{f}} \sin \alpha+\mathrm{y}_{\mathrm{f}} \cos \alpha+\mathrm{y}_{0}-\mathrm{y} .
\end{aligned}
$$

Finally, we can relate the rotated field to the one caused purely by stress release

$$
\begin{aligned}
\mathrm{u}_{\mathrm{x}}^{\prime}(\mathrm{r}, \theta)= & \left(\frac{\sigma \mathrm{R}_{0}^{2}}{\mathrm{Er}}(1+\nu) \cos \theta+\mathrm{r} \cos \theta\right) \cos \alpha \\
& +\left(\frac{\sigma \mathrm{R}_{0}^{2}}{\mathrm{Er}}(1+\nu) \sin \theta+\mathrm{r} \sin \theta\right) \sin \alpha \\
& +\mathrm{x}_{0}-\mathrm{r} \cos \theta \\
\mathrm{u}_{\mathrm{y}}^{\prime}(\mathrm{r}, \theta)= & -\left(\frac{\sigma \mathrm{R}_{0}^{2}}{\mathrm{Er}}(1+\nu) \cos \theta+\mathrm{r} \cos \theta\right) \sin \alpha \\
& +\left(\frac{\sigma \mathrm{R}_{0}^{2}}{\mathrm{Er}}(1+\nu) \sin \theta+\mathrm{r} \sin \theta\right) \cos \alpha \\
& +\mathrm{y}_{0}-\mathrm{r} \sin \theta .
\end{aligned}
$$


TABLE I

VALUES OF THE DiFFERENT PARAMETERs OBTAINED BY THE SimULTANEOUS FITTING OF DISPLACEMENT FIELDS $1 \mathrm{x}$ AND $11 \mathrm{y}$

\begin{tabular}{|c|l|}
\hline $\mathbf{S}(\sigma / \mathbf{E}(\mathbf{1}+v))$ & $0.00403 \pm 0.00001$ \\
\hline $\mathbf{x}_{\mathbf{0}}(\mathrm{nm})$ & $-5.22 \pm 0.01$ \\
\hline $\mathbf{y}_{\mathbf{0}}(\mathrm{nm})$ & $-2.05 \pm 0.01$ \\
\hline$\alpha(\mathrm{rad})$ & $-0.00022 \pm 2.10^{-6}$ \\
\hline
\end{tabular}

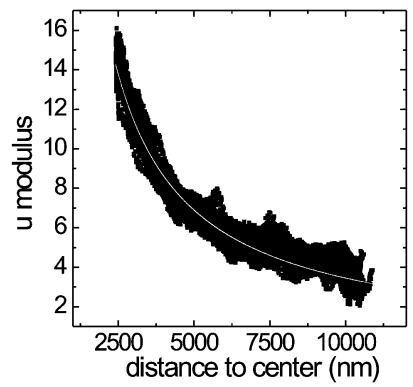

Fig. 8. Comparison between the displacement modulus of the derotated experimental field (black points) with the best fitted analytical expression (gray line).

Simultaneous fitting of both measured components $\mathrm{u}_{\mathrm{x}}^{\prime}$ and $\mathrm{u}_{\mathrm{y}}^{\prime}$ of the experimental data fields to the analytical expressions of a rotated field given in (21) and (22) were performed with $\mathrm{R}^{2}$ equal to 0.958 . In order to simplify the process and to avoid any influence of the Young modulus and the Poisson ratio accuracies, the quotient $\sigma / \mathrm{E}(1+\nu)$ has been taken as a unique fitting parameter.

Table I summarizes the obtained values for the four fitting parameters, that is, $\sigma / \mathrm{E}(1+\nu), \mathrm{x}_{0}, \mathrm{y}_{0}$, and $\alpha$. The quality of the fit can be observed in Fig. 7(c) and (d), where the contour lines of the fitted analytical displacement fields $u_{x}$ and $u_{y}$ are depicted. The estimated standard deviation of the determined ratio $\sigma / \mathrm{E}(1+\nu)$ of only $0.2 \%$ illustrates the effect of whole field fitting as opposed to a two-point measurement as considered in Section II-B. This can be again observed in Fig. 8, where the displacement modulus of the approximately 8000 derotated experimental data points and the best fitted curve have been depicted.

Once the fitting parameters are obtained, the residual stress of the structure can be extracted from

$$
\sigma=S(1+\nu) E
$$

The residual stress value obtained using the Young Modulus value of $219 \pm 15 \mathrm{GPa}$ (mean value computed from nanoindentation and bulge test results) and considering again a Poisson ratio of 0.23 corresponds to $1085 \pm 70 \mathrm{MPa}$, a value that is in agreement with findings from the bulge test (within 7\%).

\section{DISCUSSION}

In the present paper the feasibility of a novel approach to residual stress measurement with high spatial resolution has been demonstrated. The method has been tested on a silicon nitride micromachined membrane, but it can be transferred to any prestressed free-standing microstructure.

Assuming a Poisson ratio of 0.23 , a difference of $7 \%$ has been found between the stress value obtained with this new method and the one obtained by applying the "classical" bulge test method.
One of the reasons for this discrepancy can be explained by possible stress fluctuations on the membrane. That is, in the new method the stress measurement is performed locally, being the area of influence proportional to the milled radius. Therefore, the milling of a hole of $4.5 \mu \mathrm{m}$ radius modifies the stress distribution within a certain distance (at $50 \mu \mathrm{m}$ the stress has recovered $99.5 \%$ of its original value) whereas the bulge test provides an average stress value of the whole membrane, where the effects of clamping can be also playing a certain role [25].

However, another possible origin of this difference could still lie on the patterned coating required to perform the correlation analysis. Although being very thin and configured in small islands (as shown in Fig. 4), the platinum coating deposited on top of the layer could also exert some influence in the measured stress. Finally, the influence of ion milling on material constitution around the milled hole could also affect the measured stress value. These effects will have to be investigated in the future in order to discard possible sources of error in the measurement.

The fitting procedure of the 2-D data field allows to obtain the value of the quotient $\sigma / \mathrm{E}(1+\nu)$ with high accuracy even for high relative error values of single measurement points (see Table I). In this way, the accuracy in the computed residual stress value relies mainly on how accurately the mechanical parameters involved in the fitting parameter $\mathrm{S}$ (Young modulus and Poisson ratio) are known. However, the knowledge of the Poisson ratio is required not only in the residual stress value extraction but also in the Young modulus determination. That is, Poisson ratio is required both in the nanoindentation test and in the bulge test if, as happens to be in the current case, only one geometry is used. The approximation to membrane testing of Vlassak and Nix [26] eliminated the need to assume a value of Poisson ratio by testing silicon nitride films with different aspect ratios. In this particular case, as only one membrane geometry was available and no independent measurements of the Poisson ratio were available for the tested material (i.e., tensile tests), a value of 0.23 (suggested as an initial design value in [3]) was assumed in all the calculations. When doing so, the computed accuracy of the residual stress depends exclusively on the accuracy of the Young modulus and yields the relative error of $6 \%$ provided in the last section.

However, an estimation of the influence of the uncertainty in the Poisson ratio value can be given, if we take into consideration the values reported for this parameter in the literature. Among the scarce measurements reported for LPCVD silicon nitride, values as large as 0.28 can be found [26].

The introduction of this new value (a value $20 \%$ larger that the initial one) has to be carried out not only in the $(1+\nu)$ term in expression (23) but also in Young modulus computation. Thus, whereas the new Poisson ratio value generates an increase of $4 \%$ in the first term of (23), it causes the Young modulus values found in both measurement techniques to decrease. In the bulge test, the new Poisson ratio has to be introduced in $\mathrm{C}_{2}(\nu)$ coefficient (see (12)) modifying it from 2.43 to 2.56 . This variation leads to a decrease of $5 \%$ in the resultant Young modulus value. In a similar way, the expression used for the Young modulus extraction in nanoindentation measurements has a dependence with the Poisson ratio of the layer under study such as $\mathrm{E}=\left(1-\nu^{2}\right) \phi\left(\mathrm{E}_{\mathrm{r}}, \mathrm{E}_{\mathrm{i}}, \nu_{\mathrm{i}}\right)$, being $\mathrm{E}_{\mathrm{r}}$ the reduced Young modulus 
obtained from the experimental load-displacement behaviour and $\mathrm{E}_{\mathrm{i}}, \nu_{\mathrm{i}}$ the mechanical properties of the indenter. The introduction of a Poisson ratio of 0.28 in this expression results in a 3\% decrease of the Young modulus. Therefore, taking into account the most pessimist case in which the Young modulus experiences a decrease of $5 \%$, that is, it drops from $219 \pm 15$ to $209 \pm 14 \mathrm{GPa}$ and the Poisson ratio turns to be 0.28 instead of 0.23 , the resultant computed stress value changes from $1085 \pm 70$ to $1078 \pm 70 \mathrm{MPa}$, a variation of less than $1 \%$. It can hence be concluded that the existing uncertainty in the value of the membrane's Poisson ratio is not critical for the determination of its residual stress.

\section{CONCLUSION}

In this paper, a new approach to the widely known holedrilling method technique for residual stress has been presented. The combined imaging-milling features of a FIB equipment have allowed to scale down this method to the microscale and therefore to perform for the first time a highly localized measurement of residual stress of a silicon nitride micromachined membrane. This structure has been chosen to demonstrate the feasibility of this new approach for two reasons-a $\mathrm{Si}_{3} \mathrm{~N}_{4}$ membrane plays an important role as a mechanical support in a wide-range of membrane-based sensors and its residual stress can easily be characterized by means of the bulge test providing a reference value for comparison. Results obtained with this new method are consistent with the reference value. The presented methodology gives access to a completely new approach to stress measurement in MEMS structures. According to its nature, the method possesses an excellent dowscaling capability that renders it specially suitable for future applications in nanostructures.

\section{REFERENCES}

[1] W. M. van Spengen, "MEMS reliability from a failure mechanisms perspective," Microelectron. Reliab., vol. 43, pp. 1049-1060, 2003.

[2] L. B. Freund and S. Suresh, Thin Film Materials - Stress, Defect Formation and Surface Evolution. Cambridge, U.K.: Cambridge Univ. Press, 2003.

[3] M. Gas-el-Hak, The MEMS Handbook. Boca Raton, FL: CRC, 2002.

[4] J. A. Schweitz, "Mechanical characterization of thin films by micromechanical techniques," MRS Bull., pp. 34-45, Jul. 1992.

[5] H. Guckel, T. Randazzo, and D. W. Burns, "A simple technique for the determination of mechanical strain in thin films with applications to polysilicon," J. Appl. Phys., vol. 57, no. 5, pp. 1671-1674, 1985.

[6] M. Godin, V. Tabard-Cossa, P. Grutter, and P. Williams, "Quantitative surface stress measurements using a microcantilever," Appl. Phys. Lett., vol. 79, no. 4, pp. 551-553, Jul. 2001.

[7] J. Laconte, F. Iker, S. Jorez, N. Andre, J. Proost, T. Pardoen, D. Flandre, and J. P. Raskin, "Thin films stress extraction using micromachined structures and wafer curvature measurements," Microelectron. Eng., vol. 76, pp. 219-226, 2004.

[8] Determining Residual Stresses by the Hole-Drilling Strain-Gage Method, , ASTM Standard E837.

[9] G. Focht and K. Schiffner, "Determination of residual stresses by an optical correlative hole-drilling method," Experiment. Mechan., vol. 43, pp. 97-104, Mar. 2003.

[10] B. Michel and R. Kühnert, "Micro-Moire-methode und microDACverfahren anwenden," Zeitschrift Materialprufung, vol. 38, no. 6, 1996.

[11] B. Li, X. Tang, H. Xie, and X. Zhang, "Strain analysis in MEMS/NEMS structures and devices by using focused ion beam system," Sens. Actuators $A$, vol. 111, pp. 57-62, 2004.
[12] D. Vogel, A. Gollhardt, and B. Michel, "Micro and nanomaterials characterization by image correlation methods," in Sens. Actuators A, 2002, vol. 99, pp. 165-171.

[13] S. Cho, J. F. Cárdenas-García, and I. Chasiotis, "Nano-displacement based microscopic hole method for determination of thin film properties," Sens. Actuators A, vol. 120, pp. 163-171, 2005.

[14] S. W. Cho, I. Chasiotis, T. A. Friedman, and J. Sullivan, "Direct measurements of Young's modulus, Poisson's ratio and failure properties of ta-C MEMS," J. Micromech. Microeng., vol. 25, no. 4, pp. 728-735, 2005.

[15] D. Vogel, A. Gollhardt, J. Keller, and B. Michel, "fibDAC deformation measurements," in The 8th Europ. FIB Users Group Meet., Zurich, Oct. 4, 2004.

[16] M. T. Flaman, "Brief investigation of induced drilling stresses in the center hole method of residual stress measurement," Experiment. Mechan., vol. 22, pp. 26-30, Jan. 1982.

[17] D. Vogel, A. Schubert, W. Faust, R. Dudek, and B. Michel, "MicroDAC-A novel approach to measure in-situ deformation fields of microscopic scale," in Proc. ESREF '96, Enschede, Oct. 8-11, 1996, vol. 36, Microelectron. Reliab., pp. 1939-42, 11/12.

[18] M. A. Sutton, W. J. Walters, W. H. Peters, W. F. Ranson, and S. R. Neil, "Determination of displacements using an improved digital image correlation method," Image Vision Computing, vol. 1, no. 3, pp. 133-139, 1983.

[19] S. Timoshenko and J. M. Goodier, Theory of Elasticity. New York: McGraw-Hill, 1951.

[20] C. Rossi, P. Temple-Boyer, and D. Esteve, "Realization and performance of thin $\mathrm{SiO} 2 / \mathrm{SiNx}$ membrane for microheater applications," Sens. Actuators A, vol. 64, pp. 241-245, 1998.

[21] I. Simon, N. Bârsan, M. Bauer, and U. Weimar, "Micromachined metal oxide gas sensors: Opportunities to improve sensor performance," Sens. Actuators B, vol. 73, pp. 1-26, 2001.

[22] O. Tabata, K. Kawahata, S. Sugiyama, and I. Igarashi, "Mechanical property measurement of thin films using load-deflection of composite rectangular membranes," Sens. Actuators, vol. 20, pp. 135-141, 1989.

[23] D. Maier-Schneider, J. Maibach, and E. Obermeier, "A new analytical solution for the load-deflection of square membranes," J. Microelectromech. Syst., vol. 4, pp. 238-241, 1995.

[24] W. C. Oliver and G. M. Pharr, "An improved technique for determining hardness and elastic modulus using load and displacement sensing indentation experiments," J. Mater. Res. , vol. 7, no. 6, pp. 1564-1575, 1992.

[25] A. Chouaf, C. Malhaire, M. L. Berre, M. Dupeux, F. Pourroy, and D. Barbier, "Stress analysis at singular points of micromachined silicon membranes," Sens. Actuators A, vol. 84, pp. 109-115, 2000

[26] J. J. Vlassak and W. D. Nix, "A new bulge test technique for the determination of Young's modulus and Poisson's ratio of thin films," $J$. Mater. Res., vol. 7, no. 12, pp. 3242-3249, 1992.

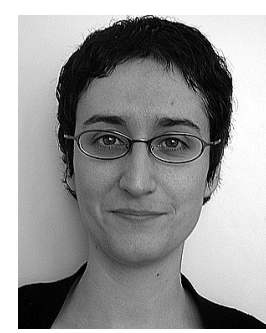

Neus Sabaté was born in Tarragona, Spain. She received the B.Sc. degree in physics from Barcelona University, Barcelona, Spain, in 1998, and the Ph.D. degree in physics in 2003, from the Microsystems Department of Centro Nacional de Microelectronica, Barcelona.

In 2004, she joined the Electronics Department, University of Barcelona, where she worked in MEMS applications for the gas sensing field. She is currently working in MEMS reliability issues and particularly in stress investigations with the Micro Materials Center, IZM Fraunhofer, Berlin.

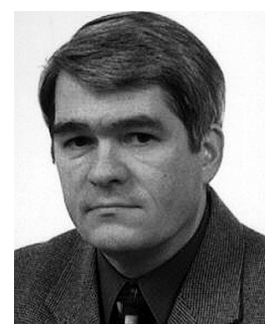

Dietmar Vogel received the Ph.D. degree in plasma physics from St. Petersburg State University, Russia, in 1980.

Since 1993, he has been working with the Fraunhofer Institute for Reliability and Microintegration Berlin (IZM).Fraunhofer, Berlin. He is Head of the Fraunhofer Nanomechanics Lab Berlin Adlershof, a division of the Micro Materials Center Berlin. His main research field is in experimental micro and nanomechanics with a special emphasis on measuring techniques. He has published approximately 100 papers, with the majority of them on mechanical reliability of electronic packaging. 


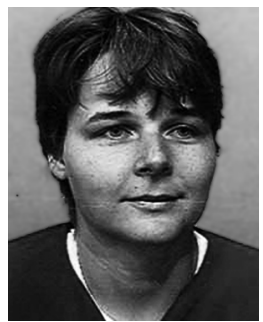

Astrid Gollhardt is a technical engineer. From 1989 to 1993 , she was staff member with the Department von Klitzing, Max Planck Institute of Solid State Research, Stuttgart. Since 1997, she has been with the Department of Mechanical Reliability and Micro Materials, Fraunhofer IZM, Berlin, Germany. Her main field of activity is focused on SEM and FIB, in situ mechanical and heating measurement in SEM and AFM, and microDAC deformation measurements in packaging.

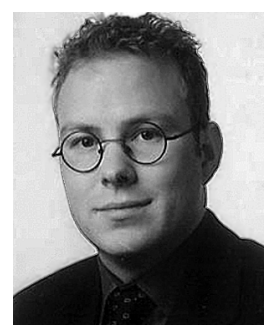

Jürgen Keller received the Diploma in mechanical engineering from the University of Kaiserslautern in 1999 , and in 2005, he received the Ph.D. degree from the Lehrstuhl Polymermaterialien, Brandenburg University of Technology Cottbus and the Fraunhofer Institute for Reliability and Micro Integration (IZM), Berlin.

Since then, he has been with Fraunhofer IZM working on micro- and nanomechanical deformation measurement techniques for microelectronics and nanotechnology applications.

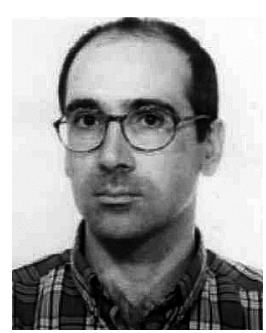

Carles Cané received the B.Sc. degree in telecommunication engineering in 1986 and the Ph.D. degree in 1989 from the Universitat Politècnica de Catalunya, Barcelona, Spain.

Since 1990, he has been Permanent Researcher with the National Microelectronics Center (CNM), Barcelona. He is currently working in the fields of sensors and microsystems and their compatibility with standard CMOS technologies.

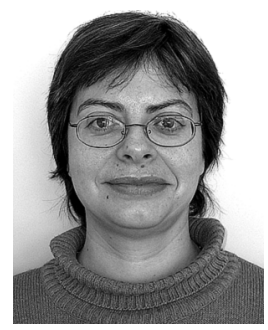

Isabel Gràcia received the $\mathrm{Ph} . \mathrm{D}$. degree in physics in 1993 from the Autonomous University of Barcelona, Barcelona, Spain, working on chemical sensors.

She joined the Microsystems Department, Centro Nacional de Microelectronica (CNM), Barcelona, where she is working on photolithography. Her work is focused on gas sensing microsystems and MEMS reliability.

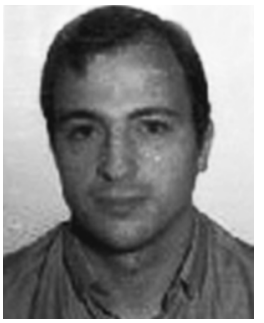

Joan R. Morante was born in Mataró, Spain. He received the Bachelor degree in 1977 and the Ph.D. degree in Physics in 1980, both from the University of Barcelona, Barcelona, Spain.

Since 1986 he has been Full Professor of Electronics and Director of the Electronic Materials and Engineering Group (EME). He is currently Head of the EME Research Group, Codirector of the Centre for Engineering of Microsystems (CEMIC) since 1999, and Director of the Centre of reference of Advanced Materials for Energy (CeRMAE ) since 2003. His activity is devoted to the electronic materials and technology, physics and chemical sensors, actuators, and microsystems. His interest is in nanoscience and nanotechnologies.

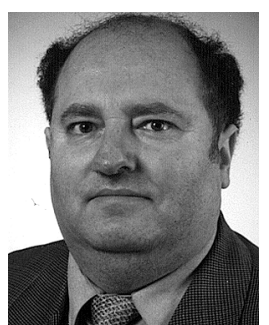

Bernd Michel received the Ph.D. degree in solid state physics from Martin-Luther-University Halle- Wittenberg in 1976.

He became Professor for Solid Mechanics with the Institute of Mechanics of Academy of Sciences Berlin, Germany, and Chemnitz in 1982. From 1993 to the present, he is Head of Department Mechanics Reliability and Micro Materials of Fraunhofer Institute IZM, Berlin, and Head of the Department of Simulation and Design of Microsystems, Technical University Berlin. Since 2000 he has also been Director of Micro Materials Center Berlin. He deals with micro- and nanoreliability concepts, fracture mechanics for microcomponents.

Dr. Michel is a recognized General Chair of International Micro Materials Conferences. 